\title{
Relationship of dysregulation of glucose metabolism with white-coat hypertension: the Ohasama study
}

\author{
Miki Hosaka ${ }^{1}$, Akira Mimura ${ }^{1,2}$, Kei Asayama ${ }^{1}$, Takayoshi Ohkubo ${ }^{1,3}$, Katsuhisa Hayashi ${ }^{4}$, Masahiro Kikuya ${ }^{1}$, \\ Michihiro Sato ${ }^{1}$, Takanao Hashimoto ${ }^{4}$, Atsuhiro Kanno ${ }^{4}$, Azusa Hara ${ }^{4}$, Taku Obara ${ }^{4,5}$, Hirohito Metoki ${ }^{6}$, \\ Ryusuke Inoue ${ }^{7}$, Haruhisa Hoshi ${ }^{8}$, Hiroshi Satoh ${ }^{9}$, Yoshitomo Oka ${ }^{10}$ and Yutaka Imai ${ }^{1}$ \\ Characteristics of glucose metabolism in subjects with white-coat hypertension (WCHT) have not been fully investigated. The \\ purpose of this study was to determine the relationship between glucose metabolism and WCHT on the basis of blood pressure \\ (BP) at home (HBP) in the general population. Participants were from Ohasama, a rural Japanese community, and included \\ 466 residents (mean age, 61.0 years) who had no history of diabetes mellitus. HBP and oral glucose tolerance test values were \\ measured. Participants were classified into four groups on the basis of their HBP and casual-screening BP (CBP) values: \\ normotension (NT) (HBP $<135 / 85 \mathrm{~mm} \mathrm{Hg}, \mathrm{CBP}<140 / 90 \mathrm{~mm} \mathrm{Hg}$ ); WCHT (HBP $<135 / 85 \mathrm{~mm} \mathrm{Hg}, \mathrm{CBP} \geqslant 140 / 90 \mathrm{~mm} \mathrm{Hg}$ ); \\ masked hypertension (HBP $\geqslant 135 / 85 \mathrm{~mm} \mathrm{Hg}, \mathrm{CBP}<140 / 90 \mathrm{~mm} \mathrm{Hg}$ ); or sustained hypertension (SHT) (HBP $\geqslant 135 / 85 \mathrm{~mm} \mathrm{Hg}$, \\ $\mathrm{CBP} \geqslant 140 / 90 \mathrm{~mm} \mathrm{Hg}$ ). The relationships between glucose metabolism and BP among the four groups were examined using \\ multivariate analysis adjusted for possible confounding factors. Factors in relation to glucose metabolism, such as fasting \\ glucose level, 2-h postchallenge glucose level and homeostasis model assessment-insulin resistance index, were significantly \\ higher in subjects with WCHT and SHT than in those with NT (all $P<0.03$ ). When men and women were analyzed separately, \\ these relationships were more pronounced in women. Our results suggest that dysregulation of glucose metabolism in WCHT \\ might contribute to the increase in the long-term cardiovascular risk among the general population. \\ Hypertension Research (2010) 33, 937-943; doi:10.1038/hr.2010.114; published online 15 July 2010
}

Keywords: general population; glucose metabolism; home blood pressure; oral glucose tolerance test; white-coat hypertension

\section{INTRODUCTION}

Self-measurement of blood pressure (BP) at home (HBP) has been recognized as a useful tool for accurate diagnosis and treatment of hypertension. Previous reports have indicated that HBP is correlated with target-organ damage, and predict the prognosis of hypertension better than casual-screening BP (CBP). ${ }^{1,2}$

The measurement of BP outside medical settings has identified a subgroup of individuals with white-coat hypertension (WCHT) ${ }^{3}$ who have persistently elevated CBP but normal HBP or ambulatory BP (ABP) levels, and a subgroup of individuals with masked hypertension $(\mathrm{MHT})^{4}$ who have normal CBP but elevated HBP or ambulatory BP levels. The clinical significance of WCHT in relation to cardiovascular disease risk is controversial., ${ }^{5,6}$ Similarly, there is little conclusive evidence about the association between WCHT and metabolic abnormalities. $^{7}$

Oral glucose tolerance test (OGTT) is widely used for diagnosing diabetes mellitus (DM). Fasting glucose level is insufficient to diagnose DM; however, measuring glucose level 2-h after an oral glucose load has strong predictive power for cardiovascular disease. ${ }^{8-10}$ Although several studies have shown the association between OGTT and $\mathrm{CBP},{ }^{11,12}$ the association between OGTT and HBP remains unclear. Moreover, the relationship of WCHT and MHT with glucose metabolism is undetermined. Therefore, the aim of this study was to determine the relationship between glucose metabolism and WCHT, as well as MHT on the basis of HBP in the general population.

\footnotetext{
${ }^{1}$ Department of Planning for Drug Development and Clinical Evaluation, Tohoku University Graduate School of Pharmaceutical Sciences, Sendai, Japan; ${ }^{2}$ Laboratory of Oncology, Pharmacy Practice and Sciences, Tohoku University Graduate School of Pharmaceutical Sciences, Sendai, Japan; ${ }^{3}$ Department of Health Science, Shiga University of Medical Science, Otsu, Japan; ${ }^{4}$ Department of Clinical Pharmacology and Therapeutics, Tohoku University Graduate School of Pharmaceutical Sciences and Medicine, Sendai, Japan; ${ }^{5}$ Department of Pharmaceutical Sciences, Tohoku University Hospital, Sendai, Japan; ${ }^{6}$ Department of Obstetrics and Gynecology, Tohoku University Graduate School of Medicine, Sendai, Japan; ${ }^{7}$ Department of Medical Informatics, Tohoku University Graduate School of Medicine, Sendai, Japan; ${ }^{8}$ Ohasama Hospital, Hanamaki, Japan; ${ }^{9}$ Department of Environmental Health Sciences, Tohoku University Graduate School of Medicine, Sendai, Japan and ${ }^{10}$ Department of Molecular Metabolism and Diabetes, Tohoku University Graduate School of Medicine, Sendai, Japan

Correspondence: Dr K Asayama, Department of Planning for Drug Development and Clinical Evaluation, Tohoku University Graduate School of Pharmaceutical Sciences, 6-3 Aramaki-aza-aoba, Aoba-ku, Sendai 980-8578, Japan.

E-mail: kei@asayama.org
}

Received 19 February 2010; revised 20 April 2010; accepted 26 April 2010; published online 15 July 2010 


\section{METHODS}

\section{Study population}

This investigation is a part of a longitudinal observational study of HBP measurements among Ohasama residents that started in 1987. The socioeconomic and demographic characteristics of this region and full details of the project have been described elsewhere. ${ }^{13}$ Between 2000 and 2008, we contacted all 4809 individuals aged $\geqslant 35$ years in four districts of Ohasama town. Those who were not at home during the normal working hours of the study nurses $(n=1298)$ and those hospitalized $(n=192)$ or incapacitated $(n=120)$ were not eligible. Of the remaining 3199 residents, 2181 (68\%) gave written, informed consent to participate in the HBP measurement program. Of those, 700 individuals (19\%) voluntarily participated in the OGTT. We excluded those treated with antidiabetic $(n=11)$ and antihypertensive agents $(n=223)$ from this analysis. The total number of participants statistically analyzed was thus 466. The study protocol was approved by the Institutional Review Board of Tohoku University School of Medicine, Sendai, Japan, and by the Department of Health of the Ohasama Town Government.

\section{BP measurement}

HBP was measured using the semi-automatic HEM-747IC-N or HEM701C (Omron Healthcare, Kyoto, Japan), a device based on the cuff-oscillometric method that generates a digital display of both systolic and diastolic BP values. ${ }^{14}$ Physicians and public health nurses instructed the participants on how to use the device and record HBP results. The participants then measured their own BP once in the morning, in the sitting position within $1 \mathrm{~h}$ after awaking and after $2 \mathrm{~min}$ of rest and recorded such measurements for 4 weeks. Although many participants measured their HBP values twice or more per occasion, we used the first value from each measurement in our analysis to exclude individual selection bias. ${ }^{15}$ HBP was defined as the mean of all measurements. The mean number of total HBP measurements was 24. CBP measurements were taken after at least $2 \mathrm{~min}$ of rest, twice consecutively, using an automatic device (HEM-907, Omron Healthcare) before OGTT. The average of two consecutive readings from each individual was used as CBP. The HBP and CBP measuring devices used in this study have been validated ${ }^{14,16,17}$ and meet the criteria established by the Association for the Advancement of Medical Instrumentation. ${ }^{18}$

\section{OGTT and other information}

OGTT was carried out using a 75-g glucose-equivalent carbohydrate load (Trelan G; Ajinomoto Pharma, Tokyo, Japan) after the fasting blood samples were collected. Blood samples were drawn at $60 \mathrm{~min}(1 \mathrm{~h})$ and $120 \mathrm{~min}(2 \mathrm{~h})$, and glucose levels and insulin were measured. Information on the use of antihypertensive, hyperlipidemic and diabetic medications at baseline was obtained from interviews conducted at the time of OGTT, from records of annual health checkups and from records of Ohasama Hospital. Serum adiponectin was measured using a latex particle-enhanced turbidimetric immunoassay (SRL, Tokyo, Japan).

\section{Classification of groups}

Participants were classified into four groups (normotension (NT), WCHT, MHT and sustained hypertension (SHT)) on the basis of their HBP and CBP levels: NT, with systolic $\mathrm{CBP}<140 \mathrm{~mm} \mathrm{Hg}$ and diastolic $\mathrm{CBP}<90 \mathrm{~mm} \mathrm{Hg}$, and systolic $\mathrm{HBP}<135 \mathrm{~mm} \mathrm{Hg}$ and diastolic $\mathrm{HBP}<85 \mathrm{~mm} \mathrm{Hg}$; WCHT, with systolic $\mathrm{CBP} \geqslant 140 \mathrm{mmHg}$ or diastolic $\mathrm{CBP} \geqslant 90 \mathrm{mmHg}$ or both, and systolic $\mathrm{HBP}<135 \mathrm{~mm} \mathrm{Hg}$ and diastolic $\mathrm{HBP}<85 \mathrm{~mm} \mathrm{Hg}$; MHT, with systolic $\mathrm{CBP}<140 \mathrm{~mm} \mathrm{Hg}$ and diastolic $\mathrm{CBP}<90 \mathrm{~mm} \mathrm{Hg}$, and systolic $\mathrm{HBP} \geqslant 135 \mathrm{~mm} \mathrm{Hg}$ or diastolic HBP $\geqslant 85 \mathrm{~mm} \mathrm{Hg}$ or both; and SHT, with systolic $\mathrm{CBP} \geqslant 140 \mathrm{~mm} \mathrm{Hg}$ or diastolic $\mathrm{CBP} \geqslant 90 \mathrm{~mm} \mathrm{Hg}$ or both, and systolic $\mathrm{HBP} \geqslant 135 \mathrm{~mm} \mathrm{Hg}$ or diastolic HBP $\geqslant 85 \mathrm{~mm} \mathrm{Hg}$ or both (Figure 1). Cutoff values were derived from several guidelines. ${ }^{19-21}$

On the basis of OGTT, subjects were classified as having DM, impaired glucose intolerance, impaired fasting glucose or normal glucose tolerance according to the World Health Organization classification ${ }^{22}$ (Figure 2).

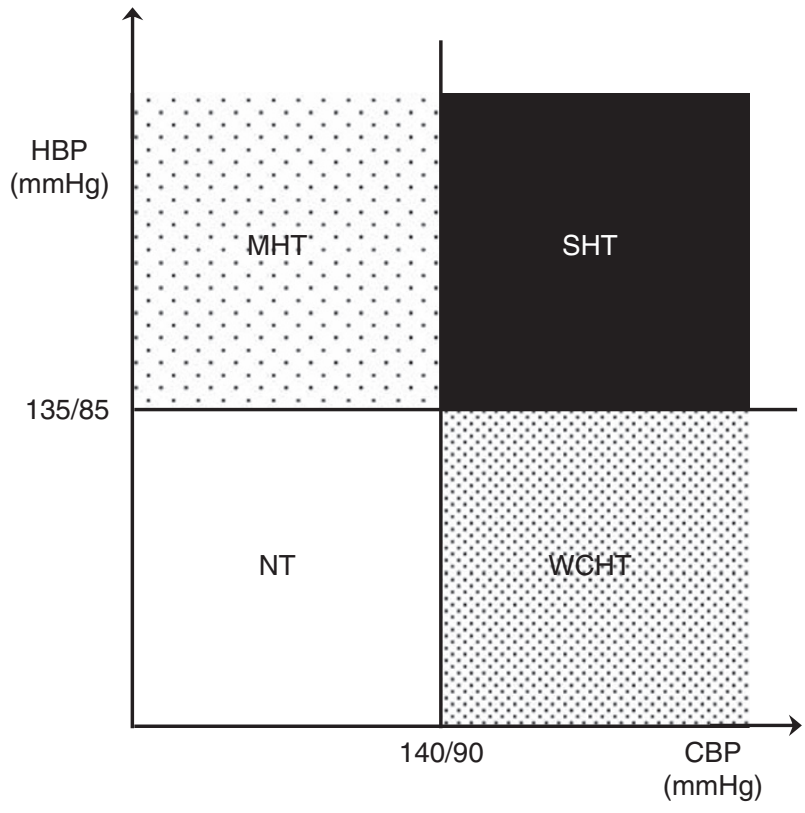

Figure 1 Distribution of subjects classified into four groups on the basis of HBP and CBP levels. CBP, casual-screening blood pressure; HBP, home blood pressure; MHT, masked hypertension; NT, normotension; SHT, sustained hypertension; WCHT, white-coat hypertension.

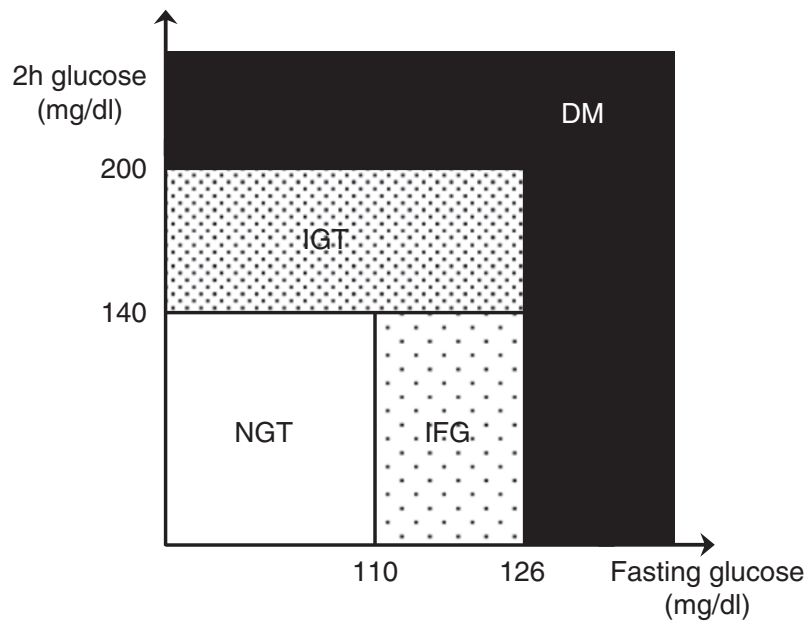

Figure 2 Distribution of subjects classified into four groups on the basis of fasting glucose and 2-h glucose level, which were determined by OGTT. DM, diabetes mellitus; IFG, impaired fasting glucose; IGT, impaired glucose tolerance; NGT, normal glucose tolerance.

\section{Data analysis}

Dyslipidemia was defined in accordance with criteria of the Japanese metabolic syndrome $\mathrm{e}^{23}$ as low high-density lipoprotein-cholesterol $(<40 \mathrm{mg}$ per $100 \mathrm{ml}$ $\left.\left(1.03 \mathrm{mmoll}^{-1}\right)\right)$, high triglyceride $\left(\geqslant 150 \mathrm{mg}\right.$ per $\left.100 \mathrm{ml}\left(1.68 \mathrm{mmoll}^{-1}\right)\right)$ and/ or the use of antilipidemic treatment. Area under the blood concentration time curve was calculated using fasting plasma glucose, 1-h glucose and 2-h glucose by quadrature by parts (area under the blood concentration time curve $=($ fasting plasma glucose $+1-h$ glucose $) \times 0.5+(1-h$ glucose +2 -h glucose $) \times 0.5)$. The homeostasis model assessment-insulin resistance index (HOMA-IR) was calculated using the following formula: HOMA-IR=fasting glucose (mg per $100 \mathrm{ml}) \times$ fasting insulin $(\mu$ Units per $\mathrm{ml}) / 405$. Insulin sensitivity was determined by the Matsuda DeFronzo index based on the following formula: 10000 / sqrt (fasting glucose $(\mathrm{mg}$ per $100 \mathrm{ml}) \times$ fasting insulin $(\mu$ Units per $\mathrm{ml})$ ) (mean glucose $(\mathrm{mg}$ per $100 \mathrm{ml}) \times$ mean insulin $(\mu$ Units per $\mathrm{ml}))^{24}$ 
All data are expressed as means \pm s.d. Variables were compared using Fisher's exact test, ANOVA (analysis of variance) or ANCOVA (analysis of covariance), followed by Tukey's multiple comparison test. Associations between indices for glucose metabolism and BPs as continuous variables were examined with multiple regression analysis adjusted by age, body mass index, dyslipidemia, history of cardiovascular disease, drinking habit and smoking habit. Statistical significance was established at $P<0.05$. All statistical calculations were carried out using the SAS system (version 9.1, SAS Institute, Cary, NC, USA).

\section{RESULTS}

The characteristics of the study participants are given in Table 1. The mean age was $61.0 \pm 9.6$ years and the proportion of men and women was 29:71. Mean systolic/diastolic CBP and HBP values were
$131.3 \pm 18.3 / 76.1 \pm 11.2 \mathrm{~mm} \mathrm{Hg}$ and $122.3 \pm 15.0 / 74.5 \pm 9.0 \mathrm{~mm} \mathrm{Hg}$, respectively. Of the 466 subjects, 268 were classified as having NT, 49 were classified as having MHT, 90 were classified as having WCHT and the remaining 59 were classified as having SHT. Both CBP and HBP values in the NT group were significantly lower those in than the other groups. Subjects in the NT group tended to be younger than those in the other categories of BP classification.

The relationships between glucose metabolism and each BP group were analyzed using ANCOVA (Table 2). Among subjects with WCHT and SHT, significantly higher glucose levels and HOMA-IR values and significantly lower Matsuda DeFronzo index values were observed when compared with NT (all $P<0.03$ ). Among those with MHT, there were no indices for glucose metabolism, which showed significant

Table 1 Characteristics of study participants

\begin{tabular}{|c|c|c|c|c|c|c|}
\hline & All subjects & Subjects with NT & Subjects with MHT & Subjects with WCHT & Subjects with SHT & ANOVA P-value \\
\hline Number of subjects $(n)$ & 466 & 268 & 49 & 90 & 59 & - \\
\hline Gender (women, \%) & 71.0 & 77.2 & 49.0 & 76.7 & 52.5 & $<0.0001$ \\
\hline Age (years) & $61.0 \pm 9.6$ & $59.8 \pm 9.7$ & $63.4 \pm 9.7$ & $62.4 \pm 8.7$ & $62.4 \pm 10.0$ & 0.02 \\
\hline Body mass index $\left(\mathrm{kg} \mathrm{m}^{-2}\right)$ & $23.3 \pm 3.2$ & $22.7 \pm 3.0$ & $24.0 \pm 3.6^{*}$ & $23.5 \pm 2.8$ & $24.9 \pm 3.6^{*, \frac{ \pm}{i}}$ & $<0.0001$ \\
\hline Height (cm) & $55.6 \pm 10.3$ & $54.1 \pm 9.2$ & $59.6 \pm 13.9$ & $54.6 \pm 8.7^{\dagger}$ & $60.2 \pm 11.6$ & 0.02 \\
\hline Weight (kg) & $154.2 \pm 8.5$ & $154.1 \pm 8.1$ & $156.8 \pm 10.4^{*}$ & $152.3 \pm 7.9^{\dagger}$ & $155.2 \pm 8.8^{*, \ddagger}$ & $<0.0001$ \\
\hline Systolic HBP (mm Hg) & $122.3 \pm 15.0$ & $114.4 \pm 10.7$ & $139.5 \pm 8.9^{*}$ & $122.5 \pm 8.5^{*, \dagger}$ & $143.4 \pm 10.3^{*, \ddagger}$ & $<0.0001$ \\
\hline Diastolic HBP (mm Hg) & $74.5 \pm 9.0$ & $70.3 \pm 6.9$ & $84.6 \pm 6.8^{*}$ & $74.3 \pm 5.7^{*, \dagger}$ & $85.4 \pm 7.1^{*, \frac{\ddagger}{\ddagger}}$ & $<0.0001$ \\
\hline Home heart rate (b.p.m.) & $65.2 \pm 7.7$ & $64.6 \pm 7.1$ & $66.9 \pm 8.1$ & $66.1 \pm 7.6$ & $64.9 \pm 9.5$ & 0.2 \\
\hline Systolic CBP (mm Hg) & $131.3 \pm 18.3$ & $120.8 \pm 12.2$ & $127.1 \pm 9.2^{*}$ & $149.4 \pm 9.1^{*, \dagger}$ & $154.5 \pm 14.4^{*, \dagger, \ddagger}$ & $<0.0001$ \\
\hline Diastolic CBP (mm Hg) & $76.1 \pm 11.2$ & $70.6 \pm 8.5$ & $75.5 \pm 7.8^{*}$ & $85.2 \pm 9.3^{*, \dagger}$ & $87.7 \pm 9.5^{*, \dagger}$ & $<0.0001$ \\
\hline HDL (mg per 100 ml) & $62.3 \pm 15.4$ & $63.0 \pm 16.0$ & $59.2 \pm 14.8$ & $62.5 \pm 14.2$ & $61.6 \pm 14.8$ & 0.4 \\
\hline Triglyceride (mg per $100 \mathrm{ml}$ ) & $100.3 \pm 62.5$ & $89.8 \pm 52.7$ & $115.6 \pm 63.5^{*}$ & $109.1 \pm 74.4$ & $122.1 \pm 73.5^{*}$ & 0.0002 \\
\hline Drinking habit (\%) & 41.9 & 40.7 & 59.2 & 26.7 & 55.9 & 0.0002 \\
\hline Smoking habit (\%) & 13.7 & 12.3 & 30.6 & 7.8 & 15.3 & 0.004 \\
\hline Dyslipidemia (\%) & 19.1 & 15.3 & 28.6 & 18.9 & 28.8 & 0.03 \\
\hline a|FG(\%) & 5.4 & 2.2 & 6.1 & 11.1 & 10.2 & 0.002 \\
\hline a & 20.0 & 17.2 & 18.4 & 24.4 & 27.1 & 0.2 \\
\hline aDiabetes mellitus (\%) & 6.9 & 3.7 & 6.1 & 8.9 & 18.6 & 0.001 \\
\hline Past history of CVD (\%) & 2.8 & 1.9 & 8.2 & 1.1 & 5.1 & 0.04 \\
\hline
\end{tabular}

Abbreviations: ANOVA, analysis of variance; CBP, casual-screening blood pressure; CVD, cardiovascular disease; HBP, home blood pressure; HDL, high-density lipoprotein; IFG, impaired fasting glucose; IGT, impaired glucose intolerance; MHT, masked hypertension; NT, normotension; SHT, sustained hypertension; WCHT, white-coat hypertension. ${ }^{\star} P<0.05$ compared with NT.

$P<0.05$ compared with MHT.

$P<0.05$ compared with WCHT.

aFG, IGT and diabetes mellitus were defined by the oral glucose tolerance test.

Table 2 Variables in relation to glucose metabolism

\begin{tabular}{|c|c|c|c|c|c|c|}
\hline & All subjects & Subjects with NT & Subjects with MHT & Subjects with WCHT & Subjects with SHT & ANCOVA P-value \\
\hline Number of subjects $(n)$ & 466 & 268 & 49 & 90 & 59 & - \\
\hline Fasting plasma glucose (mg per $100 \mathrm{ml}$ ) & $95.1 \pm 10.9$ & $93.0 \pm 9.7$ & $95.1 \pm 10.3$ & $98.7 \pm 12.3^{*}$ & $99.1 \pm 11.9 *$ & 0.0003 \\
\hline One-hour glucose (mg per $100 \mathrm{ml}$ ) & $157.1 \pm 52.6$ & $148.0 \pm 51.0$ & $156.2 \pm 44.1$ & $171.4 \pm 49.1^{*}$ & $177.7 \pm 61.4^{*}$ & 0.001 \\
\hline Two-hour glucose (mg per $100 \mathrm{ml}$ ) & $126.7 \pm 43.1$ & $119.7 \pm 37.6$ & $121.6 \pm 39.3$ & $136.8 \pm 50.1^{*}$ & $146.9 \pm 49.4^{*, \dagger}$ & 0.0007 \\
\hline Glucose $A_{U C} C_{0-120}(\mathrm{mg}$ per $100 \mathrm{ml}$ h) & $268.0 \pm 71.5$ & $254.3 \pm 67.1$ & $264.6 \pm 60.3$ & $289.2 \pm 71.0^{*}$ & $300.7 \pm 82.6^{*, \dagger}$ & 0.0002 \\
\hline$\Delta 60$ glucose $(\mathrm{mg}$ per $100 \mathrm{ml}$ h) & $62.0 \pm 48.0$ & $55.0 \pm 47.2$ & $61.0 \pm 39.3$ & $72.7 \pm 44.6^{*}$ & $78.6 \pm 56.6^{*}$ & 0.01 \\
\hline$\Delta 120$ glucose $(\mathrm{mg}$ per $100 \mathrm{ml} \mathrm{h})$ & $31.6 \pm 39.3$ & $26.7 \pm 34.5$ & $26.5 \pm 36.8$ & $38.1 \pm 45.1$ & $47.9 \pm 46.7^{*, \dagger}$ & 0.008 \\
\hline HOMA & $1.32 \pm 0.86$ & $1.20 \pm 0.71$ & $1.33 \pm 1.09$ & $1.47 \pm 0.81^{*}$ & $1.66 \pm 1.15^{*}$ & 0.03 \\
\hline MDI & $8.89 \pm 4.54$ & $9.68 \pm 4.87$ & $9.53 \pm 4.78$ & $7.25 \pm 3.13^{*, \dagger}$ & $7.26 \pm 3.54^{*, \dagger}$ & 0.0009 \\
\hline
\end{tabular}

Abbreviations: ANCOVA, analysis of covariance; $\mathrm{AUC}_{0-120}$, area under the blood concentration time curve; HOMA, homeostasis model assessment; $\mathrm{MDI}$, Matsuda DeFronzo index; MHT, masked hypertension; NT, normotension; SHT, sustained hypertension; WCHT, white-coat hypertension.

Adjusted for sex, age, body mass index, dyslipidemia, history of cardiovascular disease, drinking habit and smoking habit.

$\Delta 60$ glucose $=1$ - $\mathrm{h}$ glucose- fasting plasma glucose; $\Delta 120$ glucose $=2$ - $\mathrm{h}$ glucose-fasting plasma glucose.

$P<0.05$ compared with NT.

$\uparrow<0.05$ compared with MHT. 
Table 3 Variables in relation to glucose metabolism according to sex

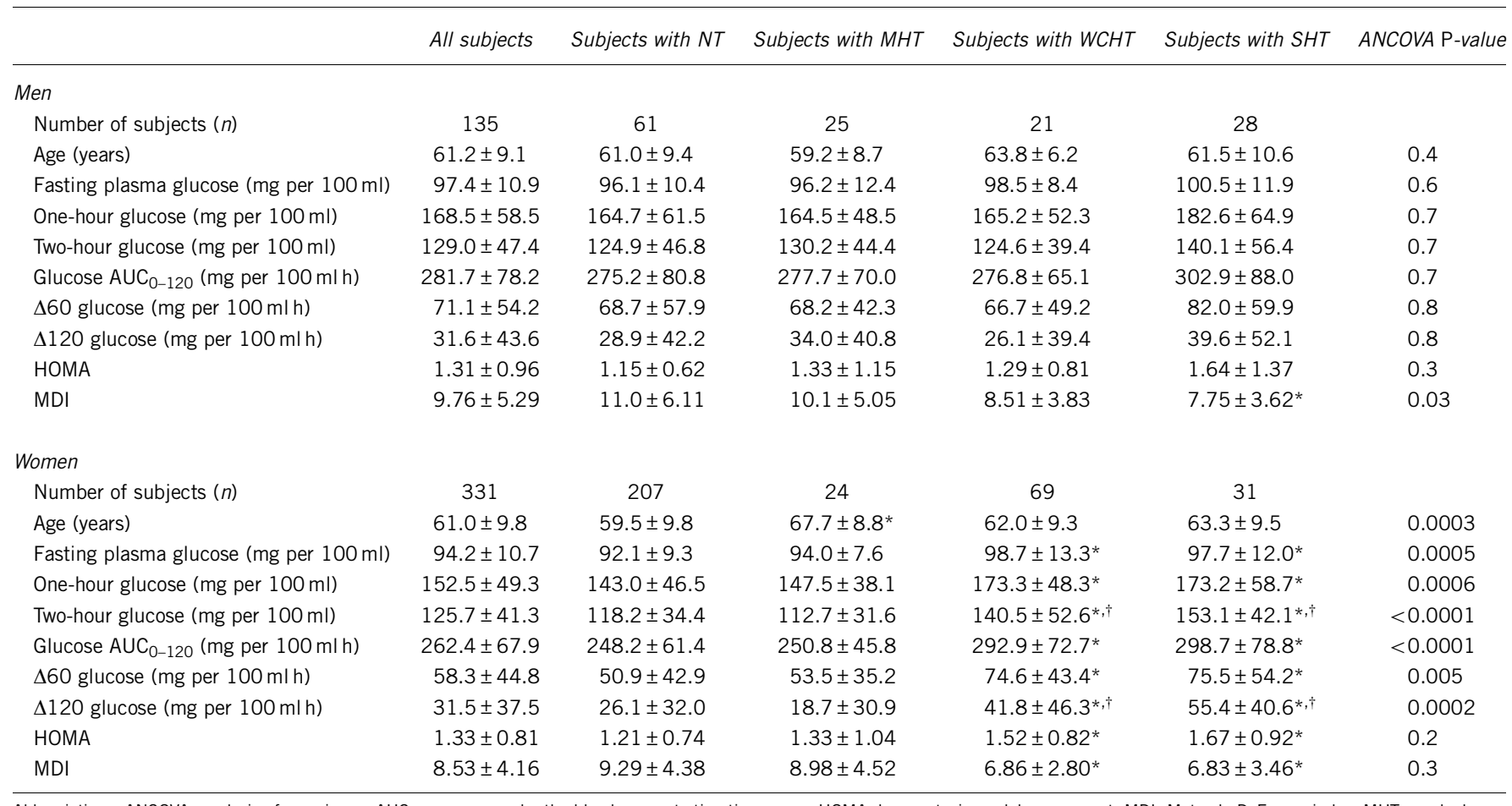

Abbreviations: ANCOVA, analysis of covariance; $\mathrm{AUC}_{0-120}$, area under the blood concentration time curve; HOMA, homeostasis model assessment; $\mathrm{MDI}$, Matsuda DeFronzo index; $\mathrm{MHT}$, masked hypertension; NT, normotension; SHT, sustained hypertension; WCHT, white-coat hypertension.

Adjusted for age, body mass index, dyslipidemia, history of cardiovascular disease, drinking habit and smoking habit.

$\Delta 60$ glucose $=1$-h glucose- fasting plasma glucose; $\Delta 120$ glucose $=2$-h glucose-fasting plasma glucose.

$P<0.05$ compared with NT

$P<0.05$ compared with $\mathrm{MHT}$.

differences from those in subjects with NT. Similarly, no significant difference was observed between subjects with WCHT and those with SHT. Further analysis in subjects in which serum adiponectin levels were measured $(n=167)$ showed that significantly lower adiponectin levels were observed in subjects with WCHT (10.5 \pm 6.0$)$ when compared with those with NT $(14.7 \pm 6.7)(P=0.044)$. Similar trends were observed only in women (data not shown).

The results in which men and women were analyzed separately are shown in Table 3. There were no significant differences in fasting glucose levels, 2-h glucose levels, HOMA-IR and the Matsuda DeFronzo index between MHT and NT regardless of sex. For women with WCHT and SHT, glucose levels were significantly higher than those with NT. Meanwhile, no significant differences of glucose levels among BP groups were observed in men. HOMA-IR in women was significantly higher in individuals with WCHT and SHT (1.5 \pm 0.8 and $1.7 \pm 0.9$, respectively) than in those with NT $(1.2 \pm 0.7)$, whereas HOMA-IR in men did not differ among the four BP groups. Similar results were observed with regard to the Matsuda DeFronzo index, although the Matsuda DeFronzo index in men was significantly higher in individuals with SHT $(7.7 \pm 3.6)$ than in those with NT $(11.0 \pm 6.1)$. However, there was no significant interaction between BP groups and sex in relation to glucose levels, HOMA-IR and the Matsuda DeFronzo index (all $P>0.2$ ).

The results of multiple regression analysis indicated that CBP values were significantly associated with several indices for glucose metabolism even adjusted by confounding factors. When systolic HBP and systolic CBP values were simultaneously included in the model (Table 4), systolic CBP, but not systolic HBP, was significantly associated with indices for glucose metabolism, especially with fasting plasma glucose $(P=0.14$ for systolic HBP, $P<0.0001$ for systolic CBP).

\section{DISCUSSION}

In this study, glucose levels in subjects with WCHT and SHT were significantly higher than those with NT. In a previous study, young subjects with WCHT tended to have metabolism dysregulation. ${ }^{25}$ In a population-based study, individuals with WCHT showed impaired insulin sensitivity compared with normotensive subjects in their late middle age. ${ }^{26}$ Sympathetic nervous system activity has been associated with the development of WCHT and with insulin resistance. $^{27,28}$ Furthermore, CBP values were reported to be positively correlated with HOMA-IR. ${ }^{29}$ Central sympathetic hyperactivity has also reported to exist in WCHT in the clinical setting. ${ }^{27}$ Although we have not investigated sympathetic nerve activities in this study, the strong relationship between CBP and glucose metabolisms would support the existence of sympathetic nervous system hyperactivity in individuals with WCHT and SHT.

In this study, significant correlations between glucose dysregulation and WCHT were not observed in men. Sympathetic nerve activity would differ between men and women with WCHT. However, to our knowledge, there was no previous study about the sympathetic nerve system for difference between men and women with WCHT. Thus we cannot explain this difference between men and women from the viewpoint of the sympathetic nerve system. Other factors would contribute to hyperglycemia in individuals with WCHT, whereas the result might be just by chance because of a small number of participants. The difference between men and women should be investigated on the basis of a large population. 
The association between WCHT and the risk of cardiovascular disease is inconsistent. Although many reports have shown that the risk of cardiovascular disease in subjects with WCHT was comparable with $\mathrm{NT},{ }^{6,30}$ our previous report indicated that WCHT is correlated with high risk for development of SHT and suggested that WCHT would carry a poor cardiovascular prognosis on a long-term basis. ${ }^{31}$ The cumulative hazard for stroke in the WCHT group was equal to that of the ambulatory hypertensive group according to the results of a meta-analysis of prospective studies, including the Ohasama study. ${ }^{32}$ Thus, dysregulation of glucose metabolism might be associated with WCHT, which is a risk factor for cardiovascular disease in the long term. Diabetic nephropathy and diabetic retinopathy were more progressive in diabetic individuals with WCHT than in those with NT. ${ }^{33}$ It would be useful for individuals with WCHT to undergo an OGTT to detect dysregulation of glucose metabolism in the early stages. Furthermore, early detection and prevention for progression from WCHT to SHT should be monitored by consecutive measurements of HBP.

Significantly low adiponectin levels were observed in subjects with WCHT compared with those with NT. The observations also support the involvement of insulin resistance in glucose dysmetabolism. The role of adipocytokine might explain sex differences for glucose metabolism as this tendency was observed especially in women; however, the number of subjects was very small especially when men and women were analyzed separately. The association between adipocytokine or sex difference and glucose metabolism should be investigated with a large number of participants.

No significant difference in indices for glucose metabolism was observed between subjects with WCHT and those with SHT. However, the tendency of low adiponectin levels was observed in subjects with WCHT compared with those with SHT. Although there were small (although not statistically significant) differences in indices for glucose metabolism between WCHT and SHT, significantly low weight and body mass index were observed in subjects with WCHT when compared with those with SHT. Despite this, the level of adiponectin in subjects with WCHT was lower and the level of glucose metabolism dysregulation was comparable when compared with those with SHT. Thus, we believe that WCHT is not comparable with SHT and might not be a safe condition.

There was no specific tendency for glucose metabolism in MHT in this study. In previous studies, fasting glucose levels were reported to be significantly higher in the MHT group than in the NT group, and those in the MHT group were similar to the SHT group. ${ }^{7,34}$ These results were inconsistent with our findings that glucose metabolism of subjects with MHT was comparable with those with NT. The most likely explanation is that individuals treated with antihypertensive medication were excluded from this study. Several previous studies in relation to the prognosis of MHT consisted of subjects treated with antihypertensive medication ${ }^{7,35}$ or included subjects both with and without antihypertensive medication. ${ }^{36}$ Although the high risk of cerebrovascular and cardiovascular disease in subjects with MHT has been established by these previous studies, the risk for individuals with MHT without antihypertensive medication would be a separate concern. Exclusion of subjects taking antihypertensive medication in the current study resulted in an insufficient number of subjects in each BP category and might lead to insufficient statistical power to draw a conclusion. Thus, further research including individuals who use antihypertensive medication would be necessary to clarify the association between MHT and dysregulation of glucose metabolism.

It should also be noted that subjects who were previously diagnosed with DM and those treated with antidiabetic agents did not participate 
in this study. Several studies including our previous study have shown that many subjects with MHT had a history of $\mathrm{DM}^{37}$ or were prescribed antidiabetic treatment. ${ }^{38,39}$ In this study, subjects with MHT might have been excessively excluded, and thus glucose levels of the MHT group might be underestimated and create a weak association between MHT and glucose levels. The possibility of selection bias should be considered when generalizing the report findings. Furthermore, the number of subjects with MHT $(n=49)$ in this study was relatively small, which resulted in an insufficient statistical power; the association between MHT and the dysregulation of glucose metabolism remain a matter for debate. It is also well known that patients with MHT have a greater frequency of targetorgan damage ${ }^{34,37}$ and have a greater risk of cardiovascular disease. ${ }^{36}$ Thus, it is important to promote further research with a large number of subjects, including those with DM to confirm the association between dysregulation of glucose metabolism and MHT.

According to the multiple regression model, CBP would be more useful for predicting dysregulation of glucose metabolism or insulin resistance than HBP. In the previous study, it was established that HBP value has a stronger predictive power for target-organ damage, morbidity and mortality than has the CBP value. ${ }^{1,2}$ Glucose metabolism is also treated as a risk factor for cardiovascular diseases. ${ }^{9,10}$ It seems reasonable to suppose that $\mathrm{HBP}$ and glucose metabolism would affect to cardiovascular diseases independently. Further followup studies are required to investigate long-term prognosis in terms of comparing BP information and glucose metabolism.

There were several limitations in this study. OGTT data were obtained at only one measurement in one occasion. If we carry out OGTT twice or more, the classification based on OGTT might be changed. We excluded patients with DM or with a history of DM. The study participants might not be the same as the entire population of Ohasama, and study participants' potential awareness of health concerns would be higher than the other residents in the general population. Thus, the possibility of selection bias needs to be considered when generalizing the present findings. Furthermore, this study included a comparably small number of men without data of participants' detailed lifestyle, although sex-specific associations were observed. Women have reported to have a greater tendency to be influenced by the white-coat effect than men, ${ }^{40,41}$ and decreased glucose tolerance related to poor lifestyle choices was more common in women than in men. ${ }^{42}$ Therefore, further prospective studies based on a sufficient number of subjects with detailed information are required to overcome these limitations.

In conclusion, strong associations between dysregulation of glucose metabolism and WCHT were observed in this study. Our findings suggest that dysregulation of glucose metabolism might contribute to the increase in the long-term risk of poor prognosis for subjects with WCHT. It is useful for individuals with WCHT to undergo OGTT to detect early stages of dysregulation of glucose metabolism. Consecutive measurements of HBP would also be important to detect and to prevent progression from WCHT to SHT.

\section{CONFLICT OF INTEREST}

The authors declare no conflict of interest.

\section{ACKNOWLEDGEMENTS}

We express our gratitude to the Ohasama public health nurses and technicians, as well as the secretarial staff of our laboratory. This study was supported in part by Grants for Scientific Research (15790293, 16590433, 17790381, 18390192, 18590587, 19590929, 19790423, 20590629, 21390201 and 21591016) from the Ministry of Education, Culture, Sports, Science, and Technology,
Japan; Grant-in-Aid (H17-Kenkou-007, H18-Junkankitou (Seishuu)-Ippan012, and H20-Junkankitou (Seishuu)-Ippan-009, 013) from the Ministry of Health, Labor and Welfare, Health and Labor Sciences Research Grants, Japan; Grant-in-Aid for Japan Society for the Promotion of Science (JSPS) fellows (16.54041, 18.54042, 19.7152, 20.7198, 20.7477 and 20.54043); Health Science Research Grants and Medical Technology Evaluation Research Grants from the Ministry of Health, Labor and Welfare, Japan; Japan Atherosclerosis Prevention Fund; Uehara Memorial Foundation; Takeda Medical Research Foundation; National Cardiovascular Research Grants; and Biomedical Innovation Grants.

1 Ohkubo T, Imai Y, Tsuji I, Nagai K, Kato J, Kikuchi N, Nishiyama A, Aihara A, Sekino M, Kikuya M, Ito S, Satoh $\mathrm{H}$, Hisamichi S. Home blood pressure measurement has as stronger predictive power for mortality than dose screening blood pressure measurement: a population-based observation in Ohasama, Japan. J Hypertens 1998; 16: 971-975.

2 Ohkubo T, Asayama K, Kikuya M, Metoki H, Hoshi H, Hashimoto J, Totsune K, Satoh H, Imai Y. How many times should blood pressure be measured at home for better prediction of stroke risk? Ten-year follow-up results from the Ohasama study. J Hypertens 2004; 22: 1099-1104.

3 Pickering TG, James GD, Boddie C, Harshfield GA, Blank S, Laragh JH. How common is white coat hypertension? JAMA 1988; 259: 225-228.

4 Pickering TG, Davidson K, Gerin W, Schwartz JE. Masked hypertension. Hypertension 2002; 40: 795-796.

5 Gustavsen PH, Hoegholm A, Bang LE, Kristensen KS. White coat hypertension is a cardiovascular risk factor: a 10-year follow-up study. J Hum Hypertens 2003; 17: 811-817.

6 Kario K, Shimada K, Schwartz JE, Matsuo T, Hoshide S, Pickering TG. Silent and clinically overt stroke in older Japanese subjects with white-coat and sustained hypertension. J Am Coll Cardiol 2001; 38: 238-245.

7 Mancia G, Bombelli M, Facchetti R, Madotto F, Quarti-Trevano F, Grassi G, Sega R. Increased long-term risk of new-onset diabetes mellitus in white-coat and masked hypertension. J Hypertens 2009; 27: 1672-1978.

8 Rodriguez BL, Lau N, Burchfiel CM, Abbott RD, Sharp DS, Yano K, Curb JD. Glucose intolerance and 23-year risk of coronary heart disease and total mortality: the Honolulu Heart Program. Diabetes Care 1999; 22: 1262-1265.

9 Tominaga M, Eguchi H, Manaka H, Igarashi K, Kato T, Sekikawa A. Impaired glucose tolerance is a risk factor for cardiovascular disease, but not impaired fasting glucose. The Funagata Diabetes Study. Diabetes Care 1999; 22: 920-924.

10 Nakagami T, DECODA Study Group. Hyperglycaemia and mortality from all causes and from cardiovascular disease in five populations of Asian origin. Diabetlogia 2004; 47: 385-394.

11 Zhang Y, Lee ET, Devereux RB, Yeh J, Best LG, Fabsitz RR, Howard BV. Prehypertension, diabetes, and cardiovascular disease risk in a population-based sample: the Strong Heart Study. Hypertension 2006; 47: 410-414.

12 Korhonen P, Aarnio P, Saaresranta T, Jaatinen P, Kantola I. Glucose homeostasis in hypertensive subjects. Hypertension 2008; 51: 945-949.

13 Imai Y, Satoh H, Nagai K, Sakuma M, Sakuma H, Minami N, Munakata M, Hashimoto J, Yamagishi T, Watanabe N, Yabe T, Nishiyama A, Nakatsuka H, Koyama H, Abe K. Characteristic of a community-based distribution of home blood pressure in Ohasama in northern Japan. J Hypertens 1993; 11: 1441-1449.

14 Imai Y, Abe K, Sasaki S, Minami N, Munakata M, Sakuma H, Hashimoto J, Sekino H, Imai K, Yoshinaga K. Clinical evaluation of semiautomatic and automatic devices for home blood pressure measurement: comparison between cuff-oscillometric and microphone methods. J Hypertens 1989; 7: 983-990.

15 Imai Y, Otsuka K, Kawano Y, Shimada K, Hayashi H, Tochikubo O, Miyakawa M, Fukiyama K. Japanese society of hypertension (JSH) guidelines for self-monitoring of blood pressure at home. Hypertens Res 2003; 26: 771-782.

16 Imai Y, Nishiyama A, Sekino M, Aihara A, Kikuya M, Ohkubo T, Matsubara M, Hozawa A, Tsuji I, Ito S, Satoh H, Nagai K, Hisamichi S. Characteristics of blood pressure measured at home in the morning and in the evening: the Ohasama study. $J$ Hypertens 1999; 17: 889-898.

17 White WB, Anwar YA. Evaluation of the overall efficacy of the Omron office digital blood pressure HEM-907 monitor in adults. Blood Press Monit 2001; 6: 107-110.

18 Association for the Advancement of Medical Instrumentation. American National Standards for Electronic or Automated Sphygmomanometers. AAMI Analysis and Review: Washington, DC, 1987.

19 European Society of Hypertension-European Society of Cardiology Guidelines Committee. 2003 European Society of Hypertension-European Society of Cardiology guidelines for the management of arterial hypertension. J Hypertens 2003; 21: 1011-1053.

20 Chobanian AV, Bakris GL, Black HR, Cushman WC, Green LA, Izzo Jr JL, Jones DW, Materson BJ, Oparil S, Wright Jr JT, Roccella EJ. The Seventh Report of the Joint National Committee on Prevention, Detection, Evaluation, and Treatment of High Blood Pressure: the JNC 7 report. JAMA 2003; 289: 2560-2572.

21 Japanese Society of Hypertension. Japanese Society of Hypertension guidelines for the management of hypertension (JSH 2004). Hypertens Res 2006; 29: S1-S105.

22 Alberti KG, Zimmet PZ. Definition, diagnosis and classification of diabetes mellitus and its complications. Part 1: diagnosis and classification of diabetes mellitus provisional report of a WHO consultation. Diabet Med 1998; 15: 539-553. 
23 Committee to Evaluate Diagnostic Standards for Metabolic Syndrome. Definition and the diagnostic standard for metabolic syndrome [in Japanese]. Nippon Naika Gakkai Zasshi 2005; 94: 794-809.

24 Matsuda M, DeFronzo RA. Insulin sensitivity indices obtained from oral glucose tolerance testing: comparison with the euglycemic insulin clamp. Diabetes Care 1999; 22: 1462-1470.

25 Julius S, Mejia A, Jones K, Krause L, Schork N, van de Ven C, Johnson E, Petrin J, Sekkarie MA, Kjeldsen SE. 'White coat' versus 'sustained' borderline hypertension in Tecumseh, Michigan. Hypertension 1990; 16: 617-623.

26 Björklund K, Lind L, Vessby B, Andrén B, Lithell H. Different metabolic predictors of white-coat and sustained hypertension over a 20 -year follow-up period: a populationbased study of elderly men. Circulation 2002; 106: 63-68.

27 Smith PA, Graham LN, Mackintosh AF, Stoker JB, Mary DA. Sympathetic neural mechanisms in white-coat hypertension. J Am Coll Cardiol 2002; 40: 126-132.

28 Esler M, Straznicky N, Eikelis N, Masuo K, Lambert G, Lambert E. Mechanisms of sympathetic activation in obesity-related hypertension. Hypertension 2006; 48: 787-796.

29 Hirose H, Saito I, Kawabe H, Saruta T. Insulin resistance and hypertension: seven-year follow-up study in middle-aged Japanese men (the KEIO study). Hypertens Res 2003; 26: 795-800.

30 Khattar RS, Senior R, Lahiri A. Cardiovascular outcome in white-coat versus sustained mild hypertension: a 10-year follow-up study. Circulation 1998; 98: 1892-1897.

31 Ugajin T, Hozawa A, Ohkubo T, Asayama K, Kikuya M, Obara T, Metoki H, Hoshi H, Hashimoto J, Totsune K, Satoh H, Tsuji I, Imai Y. White-coat hypertension as a risk factor for the development of home hypertension: the Ohasama study. Arch Intern Med 2005; 165: 1541-1546.

32 Verdecchia P, Reboldi GP, Angeli F, Schillaci G, Schwartz JE, Pickering TG, Imai Y, Ohkubo T, Kario K. Short- and long-term incidence of stroke in white-coat hypertension. Hypertension 2005; 45: 203-208.

33 Kramer CK, Leitão CB, Canani LH, Gross JL. Impact of white-coat hypertension on microvascular complications in type 2 diabetes. Diabetes Care 2008; 31: 2233-2237
34 Liu JE, Roman MJ, Pini R, Schwartz JE, Pickering TG, Devereux RB. Cardiac and arterial target organ damage in adults with elevated ambulatory and normal office blood pressure. Ann Intern Med 1999; 131: 564-572.

35 Bobrie G, Chatellier G, Genes N, Clerson P, Vaur L, Vaisse B, Menard J, Mallion JM. Cardiovascular prognosis of 'masked hypertension' detected by blood pressure self-measurement in elderly treated hypertensive patients. JAMA 2004; 291: $1342-1349$.

36 Ohkubo T, Kikuya M, Metoki H, Asayama K, Obara T, Hashimoto J, Totsune K, Hoshi H, Satoh H, Imai Y. Prognosis of 'masked' hypertension and 'white-coat' hypertension detected by 24-h ambulatory blood pressure monitoring 10-year follow-up from the Ohasama study. J Am Coll Cardiol 2005; 46: 508-515.

37 Hara A, Ohkubo T, Kikuya M, Shintani Y, Obara T, Metoki H, Inoue R, Asayama K, Hashimoto T, Harasawa T, Aono Y, Otani H, Tanaka K, Hashimoto J, Totsune K, Hoshi H, Satoh H, Imai Y. Detection of carotid atherosclerosis in individuals with masked hypertension and white-coat hypertension by self-measured blood pressure at home: the Ohasama study. J Hypertens 2007; 25: 321-327.

38 Marchesi C, Maresca AM, Solbiati F, Franzetti I, Laurita E, Nicolini E, Gianni M, Guasti L, Marnini P, Venco A, Grandi AM. Masked hypertension in type 2 diabetes mellitus. Relationship with left-ventricular structure and function. Am J Hypertens 2007; 20: 1079-1084.

39 Ben-Dov IZ, Ben-Ishay D, Mekler J, Ben-Arie L, Bursztyn M. Increased prevalence of masked blood pressure elevations in treated diabetic subjects. Arch Intern Med 2007; 22: 2139-2142.

40 Hozawa A, Ohkubo T, Nagai K, Kikuya M, Matsubara M, Tsuji I, Ito S, Satoh H, Hisamichi S, Imai Y. Factors affecting the difference between screening and home blood pressure measurements: the Ohasama Study. J Hypertens 2001; 19: 13-19.

41 Staessen JA, O'Brien ET, Atkins N, Amery AK. Short report: ambulatory blood pressure in normotensive compared with hypertensive subjects. The Ad-Hoc Working Group. J Hypertens 1993; 11: 1289-1297.

42 Dunstan DW, Salmon J, Healy GN, Shaw JE, Jolley D, Zimmet PZ, Owen N, AusDiab Steering Committee. Association of television viewing with fasting and 2-h postchallenge plasma glucose levels in adults without diagnosed diabetes. Diabetes Care 2007; 30: 516-522. 